\title{
Remote patient monitoring: a comprehensive study
}

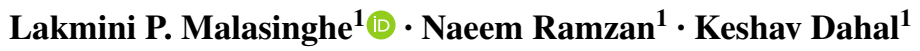

Received: 5 December 2016 / Accepted: 15 October 2017 / Published online: 26 October 2017

(C) The Author(s) 2017. This article is an open access publication

\begin{abstract}
Healthcare is a field that is rapidly developing in technology and services. A recent development in this area is remote monitoring of patients which has many advantages in a fast aging world population with increasing health complications. With relatively simple applications to monitor patients inside hospital rooms, the technology has developed to the extent that the patient can be allowed normal daily activities at home while still being monitored with the use of modern communication and sensor technologies. Sensors for monitoring essential vital signs such as electrocardiogram reading, heart rate, respiration rate, blood pressure, temperature, blood glucose levels and neural system activity are available today. Range of remote healthcare varies from monitoring chronically ill patients, elders, premature children to victims of accidents. These new technologies can monitor patients based on the illness or based on the situation. The technology varies from sensors attached to body to ambient sensors attached to the environment and new breakthroughs show contactless monitoring which requires only the patient to be present within a few meters from the sensor. Fall detection systems and applications to monitor chronical ill patients have already become familiar to many. This study provides a review of the recent advances in remote healthcare and monitoring in both with-contact and contactless methods. With the review, the authors discuss some issues
\end{abstract}

Lakmini P. Malasinghe

Lakmini.Arachchige@uws.ac.uk

Naeem Ramzan

Naeem.Ramzan@uws.ac.uk

Keshav Dahal

Keshav.Dahal@uws.ac.uk

1 School of Engineering and Computing, University of the West of Scotland, Paisley PA1 2BE, UK available in most systems. The paper also includes some directions for future research.

Keywords Remote patient monitoring $\cdot$ Mobile health . e-Health $\cdot$ Sensors $\cdot$ Wireless sensor networks

\section{Introduction}

Remote healthcare is an emerging research field as the world moves towards remote monitoring, real-time and fast detection of illnesses. Remote healthcare has many categories, (e.g. telehealth, mobile health) all of which mean monitoring of patients outside hospital conditions by the means of technology. The advantages of remote monitoring of patients are: early and real-time detection of illnesses, ability to continuously monitor patients, prevention of worsening of illnesses and untimely deaths, cost reduction in hospitalizations, reduce the number of hospitalizations, obtain more accurate readings while permitting usual daily activities for patients, improve efficiency in healthcare services by utilizing communication technology, emergency medical care, service for patients with mobility issues, emergency care for traffic accidents and other injuries and usage of non-invasive medical interventions.

Remote monitoring of patients target several sub-groups of patients, such as patients diagnosed with chronic illnesses, patients with mobility issues, or other disability, post-surgery patients, neonates and elderly patients. All these types of patients have conditions that are better to be monitored continuously. The aim of good healthcare is the ability to support ordinary life as much comfortable as possible to all patients. Most research follow the policy of allowing the mobility and activity freedom at home or personal environments which are beneficial for the patients rather than being 
confined into a high-cost hospital room. Therefore, whole systems are being built to support this concept with the use of different technologies. With the new remote health monitoring applications, elderly patients can engage in daily activities without support from a caretaker. So, these applications support activities like sitting, standing, using the bathroom, watching television, reading and sleeping, with least inconvenience to the user. Even if there are wearable sensors, these pose minimum effect to the activities. One such example is smart wrist-watch based sensors.

For the patients of accidents and sudden injuries, the time they are monitored remotely could be only the time they are being transferred to the hospital in an ambulance. Nevertheless, efforts have been focused on safe journey to the hospital and remote monitoring helps for immediate medical interventions for highly critical cases. The doctors can monitor the deterioration or maintenance of the patient while also advising the paramedics who are physically with the patients as necessary.

Basic elements of a remote monitoring system are data acquisition system, data processing system, end-terminal at the hospital and the communication network. Data acquisition system is composed of different sensors or devices with embedded sensors with data transmission capability wirelessly. With the advancement of technology, sensors may not be medical sensors only; it could be cameras or smartphones. This is because, very recent research look into contactless methods where the devices do not touch the body of the patient (McDuff et al. 2015). A most common form of these sensors used in with-contact methods are wireless sensor networks (WSN). These could be further categorized as wireless body area networks (WBAN), body area networks (BAN) or personal area networks (PAN). Data processing system includes a system with data receiving and transmitting capability and a processing unit/circuitry. Terminal at the hospital side can be either a computer (or a database) at the hospital, a dedicated device or the Smartphone of the doctor. Underlying communication network connects the data acquisition system to data processing system and further transmits the detected data and conclusions to a healthcare professional who is connected to the system via the communication network. Based on the complexity of the situation, the patient is either prompted to admit to a hospital (by sending an ambulance etc.), do certain first-aid/ caution steps and/or take certain medicines. The available remote health monitoring systems, their technologies, capabilities and actions vary to a large degree. Online databases show that the research in this area have increased in number (Fig. 1). It can be observed that already by mid-2016 the number of research results since 2014 is close to the total number of research during the previous three year period (2011-2013).

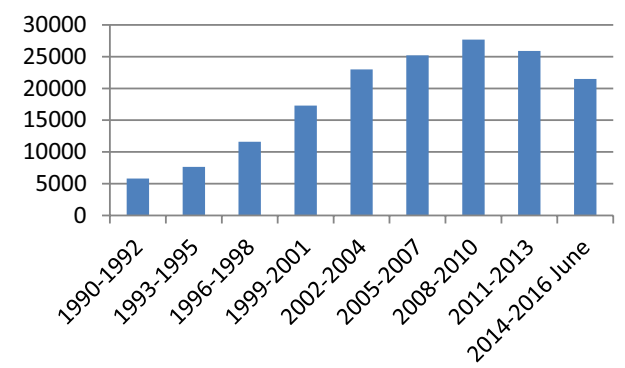

Fig. 1 Search results in Google Scholar

When it comes to designing a remote patient monitoring system, several challenges exist. Choice of whether to use with-contact or contactless method, choice of sensors, processing algorithm and communication network are the main decisions to be made when designing a remote monitoring system. Obtaining accuracy and reliability is another challenge. Receiving the approval of medical community and the consent of the patients is imperative. This study is motivated by above challenges and the importance of these systems to the general public.

With the increasing research, surveys in this field are important for new researchers as they provide an overview of existing technology. Most survey papers discuss the technology in the past two decades. This paper discusses the most recent development in remote health monitoring field from 2012 to 2016. The survey papers in this field of technology present many existing work and discuss issues in these systems, for example (Chen et al. 2014) and (Mainanwal et al. 2015). The difference of this paper is, it discusses technology, algorithms and overall systems from recent literature. Also the existing survey/review papers mainly cover withcontact methods and systems. To the best of our knowledge, there is no survey paper on contactless remote monitoring systems that analyse the studies done during 2012-2016, under the same targeted diseases discussed here. This paper aims to fulfil the requirement of a survey paper that analyses both with-contact and contactless, image-based remote health monitoring systems from very recent literature i.e. developed/proposed between 2012 and 2016.

Therefore, the contributions of this paper are;

- Discussion of with-contact remote health monitoring systems in the time period 2012-2016

- Analysis of contactless image-based methods in remote patient monitoring in the time period 2012-2016

- Discuss other applications related to remote monitoring systems

This paper is organized as follows: Sect. 2 presents most recent reviews on remote patient monitoring methods and different technologies. Section 3 provides a detailed review 
of significant studies categorised based on the targeted disease with a further subdivision based on the method of data acquisition (with-contact or contactless). Section 4 includes a learning outcome based on technologies in each different category and some insights into future research directions. Section 5 concludes the paper.

\section{Remote patient monitoring}

Remote patient monitoring systems are designed to obtain a number of physiological data from patients. Most common data are Electrocardiogram (ECG), Electroencephalogram (EEG), heart beats and respiration rate, oxygen volume in blood or pulse oximetry, signals from the nervous system, blood pressure, body/skin temperature and blood glucose level. In addition to these, sometimes, weight of the patient, level of activity of the patient and sleep data are collected. A number of researches have been done for wound management and sleep monitoring applications.

Traditional systems collect data using sensors attached to the body. But these systems pose difficulty in terms of mobility for the patient and a patient's activities in daily living. Since this kind of devices influence patient's comfort, sensitive physiological data get influenced. So the readings may not represent a patient's actual illness but rather the discomfort a patient, especially an elderly patient, is undergoing at the time a physiological reading is being taken. While there are many situations that invasive or with -contact interventions are necessary, novel research look into methods for obtaining physiological data as non-invasively as possible. For this end, contactless methods are being researched on extensively during the past few years. In this paper we discuss both with-contact (i.e. contact-based) and contactless methods as both these types of systems are important for a researcher in the field.

The remainder of this section briefly lists past general reviews on remote health monitoring systems. Disease-specific reviews are given in the Sect. 3 under each disease type.

General surveys on contact-based RPM systems Remote patient monitoring concept first came up with contact-based methods. These systems use different sensors, processing technologies, communication technologies, post-processing actions, databases and receivers/end-terminals. They also target different diseases. The variety in systems may exist due to the designer's choice or the desired outcome of other special features (embedded, low cost, low power etc.) the system designer planned for its users. For example, the designer may have been targeting a system with increased power efficiency or medication management.

This section gives an introduction on recent survey papers on contact-based remote monitoring systems.
In the review paper (Silva et al. 2015), developments in Mobile Health (m-Health) and mobile health applications in the market are discussed. This paper reviews papers on applications such as foetal heart rate monitor using mobile phone, medical health record system for paediatric human immunodeficiency virus (HIV), haemoglobin meter, mobile phone image transmission for diagnosis in addition to typical applications such as pulse oximeter. Reviews on two studies which use serious gaming approaches for learning ultrasound needle placement skills and collaborative exergaming applications for child obesity epidemic using peer-to-peer architectures have been given (Silva et al. 2015). Radio Frequency Identification (RFID) positioning, bio-patch and system-on-chip systems, cognitive stimulation through digital TV, ViCare (Hossain and Ahmed 2012) — an elderly people monitoring and virtual reality based surgical simulator are some significant work discussed in this paper. Additionally, it reviews a few papers based on technological improvements of WBSN and Wi-Max and ICT platforms generally.

In survey paper (Sawand et al. 2015) security issues in wireless body area networks and some existing solutions are discussed. It describes eHealth systems to have three key usages such as the patients, medical service providers and health professionals. They present architecture of eHealth systems with layers in terms of functionality. The paper also stresses on the importance of different security aspects such as patient's information, physiological data recordings and about the patient's environment. In a remote monitoring system, these data will be obtained by sensors and the sensor system together with other system components will need to have advanced security and privacy protection features in order to face any malicious attacks. Any malicious attack can harm the software as well as the functionality of the hardware which will pose a greater threat to the patient. Also emphasis is given on ensuring safe device-to-device communication and further data transmission (beyond the WBAN). Some important remedies for these issues have been suggested as role based access control, use of data based on accountability and revocability in case of a malicious attack. For the security in the communication and network level, secure routing protocols, cipher based algorithms and digital signature usage are presented. For the transmission of information beyond the personal area network, the paper suggests data encryption, data integrity, freshness protection and authentication. In Sawand et al. (2015) some existing solutions are given for data storage related security breaches and data access related security breaches. For the former case, they show the findings of another research where data is encrypted and divided into a number of blocks which will be saved in a number of different nodes. The data blocks will have algebraic signatures which will be useful for identifying the data. This method has been understood as to have low computational overhead. For the data access 
related security breaches, good countermeasures shown are symmetric key cryptography based schemes and public key cryptography based schemes. Biometric cryptography using key management for the inter-WBAN communications are also presented. Use of advanced image and signal processing is also suggested.

Another important aspect of this paper is to discuss energy management in WBANs. Power-saving hardware and light weight routing algorithms are suggested. Usage of hybrid encryption techniques like elliptic curve cryptography has been cited in Francis et al. (2015). This further speaks about weighted user access control, other encryption methods and data encryption such as set partitioning in hierarchical trees (SPIHT) based encryption method used for encrypting ECG signals. Further, a security architecture for eHealth systems is presented in this paper.

The survey paper (Chen et al. 2014) is on advancement of machine to machine communications in healthcare, energy management and entertainment applications. According to the paper, machine to machine communication systems are composed of three domains; namely, machine to machine device domain, network domain and application domain. The device domain is where the data collection, processing and issues like power saving of the devices will be considered. In the network domain, making compatible network between large co-existing points are addressed while in the application domain various monitoring, controlling and management services are focused. Then, the remaining discussion of the paper has been focused to wireless body area subnetworks and use of mobile cloud computing in the area of remote healthcare. Some of the commercially available sensors are given here such as accelerometer/ gyroscope, wireless pulse oximeter sensor, electromyogram (EMG) sensor, electroencephalogram (EEG) sensor, various environmental status monitoring sensors (such as carbondioxide- $\mathrm{CO}_{2}$ sensor, humidity, temperature sensor), infrared (IR) sensors, occupancy and motion detection sensors, and power sensors. Most importantly, various important issues in machine to machine communications; interference, channel dynamics, resource constraints, device heterogeneity, selforganization, quality of service (QoS) Support and Security are also raised in the paper.

Security issues in WBANs, 'the necessity of secure and dependable distributed data storage, and fine grained distributed data access control' are discussed in the review paper (Mainanwal et al. 2015). It also discusses the importance of the following: data confidentiality, data integrity, data authentication, data freshness, secure localization, availability and secure management.

Another recent brief survey (Tsakalakis and Bourbakis 2014) compares some existing systems. Recent work based on wearable sensor based systems, implantable sensor systems and portable and implantable biosensor based systems are compared.

A lot of important developments in the field such as cuffless blood pressure meter, wrist-watch based fall detection method and cane-based fall detection system are reviewed in Patel et al. (2012). Some existing systems for infant monitoring are analysed in Zhu et al. (2015). In the paper (Hu et al. 2013), discussions about photoplethysmography technology and some comparisons between these systems with existing with-contact methods are done.

General surveys on contactless RPM systems Contactless remote health monitoring methods can be categorised mainly into two sections; image-based methods and radar-based methods. Image-based systems analyse images of patients as their data and detect illnesses or falls, radar based methods used radio frequencies to get inputs for their systems and also sometimes have patient localization capability. These systems are comparatively new (only few years since inception) so there are a lot of areas for improvement. Although it is the case, some works of research have achieved good outcomes. The remainder of this section discusses some of the very recent reviews on contactless image-based patient monitoring methods.

McDuff et al. (2015) review photoplethysmographic imaging methods for non-contact remote health monitoring. This technique uses camera colour signals that are processed to recover the blood volume pulse signal out of which physiological data such as pulse rate, pulse rate variability, respiration rate and blood oxygen levels can be obtained.

A recent survey gives an insight to the studies carried out in vision based patient monitoring (Sathyanarayana et al. 2015). They present the analysis in seven different applications (including detection of illnesses, sleep monitoring and activity monitoring) and the advancement of vision-based patient monitoring technology for these applications. Use of single cameras, multi-camera arrangements, infrared cameras, time-of-flight cameras and other sensors such as bio-inspired vision sensors and monocular vision sensors is discussed.

Baig and Gholamhosseini (2013) compare several smart health monitoring systems and discuss challenges and issues in the current systems. This paper analyses the advancements of health monitoring systems under two categories; smart systems and traditional systems. Smart systems include remote health monitoring systems, mobile health monitoring systems and wearable health monitoring systems while traditional category is on wired health monitoring systems (i.e. general health monitoring systems).

Erden et al. (2016) has done a very recent survey on sensors used in assisted living. In this review, the focus is on signal and image processing methods used in recent systems. They point out that assisted living systems should be low cost, with high accuracy and user acceptance while there 
should also be mechanisms for better privacy and protection of data while sharing the minimal private data essential for being monitored remotely. The review also stresses that machine-learning algorithms customized for sensors should be used as there are a number of various sensors (passive infrared sensors, vibrational and acoustic sensors, cameras, etc.) used in a system for assisted living. Several algorithms are used for these multi-sensor systems. These algorithms include Discrete Fourier Transform, Discrete Wavelet Transform, complex wavelet transform techniques etc. Classifiers mentioned in the review include machine learning methods such as support vector machines and k-nearest neighbour methods.

With the above reviews available in the literature, the authors of this paper review the studies during the period 2012-2016. A few very recent studies (from 2017) have also been included considering their importance for a reader who would pursue further research in this area. As mentioned before, the systems are categorized based on the targeted disease. We chose only four categories based on the criticality and prevalence of them in the present society. As such, the scope of this paper is limited to the following four categories which will be discussed in detail in Sect. 3.

1. Cardiovascular System and Respiratory System related diseases

2. Fall detection and Mobility related diseases

3. Brain, Neurological Disorders and Mental Health

4. Diabetes

The above four sections were decided to be included in the view that most of the patients monitoring technologies target the ailments that fall under the above four. In each

Table 1 Research articles on various remote patient monitoring systems

Cardiogory

Cardiovascular system and respiratory system related diseases

Fall detection and mobility related diseases monitoring systems

Brain, Neurological Disorders and Mental Health

Diabetes

Other related applications

\section{References}

Szydlo and Koneiczny (2015), Kozlovszky et al. (2015), Ricci et al. (2013), Ramesh et al. (2012), Ling et al. (2015), Kumar and Kotnana (2012), Otoom et al. (2015), Thelen et al. (2015), Bisio et al. (2015a, b), Papon et al. (2015), Donnelly et al. (2015), Mishra and Agrawal (2015), Pinheiro et al. (2013), Karlen et al. (2014), Tanantong et al. (2015), Bifulco et al. (2014), Luprano et al. (2013), Thakur and Anoop (2015), Malhi et al. (2012), Bogdan et al. (2015), Monkaresi et al. (2014), Lukáč et al. (2014), Eastwood-Sutherland et al. (2014), Chandrasekar et al. (2012), Parnandi and Gutierrez-Osuna (2013), Alzahrani and Whitehead (2015), Colantonio et al. (2015), González-López et al. (2015), Mishra et al. (2015), Ganesan et al. (2015), Shao et al. (2016), Pereira et al. (2013), Couderc et al. (2014), Sugita et al. (2015), Rodríguez and Castro (2015), Janssen et al. (2016), Alves et al. (2015), Hassan et al. (2017), Sharma et al. (2017), Tarassenko et al. (2014) and Elfaramawy et al. (2017)

Sannino et al. (2015), Wang et al. (2013), Gibson et al. (2016), Paoli et al. (2012), Naranjo-Hernandez et al. (2012), Cao et al. (2012), Yu et al. (2012), Bai et al. (2012), Mastorakis and Makris (2014), Mubashir et al. (2013), Khan and Hoey (2017), Greene et al. (2016) and Cippitelli et al. (2017)

Adams et al. (2017), Lanata et al. (2015), Rasmussen et al. (2015), Fioravanti et al. (2015), Nadeem et al. (2015), Karan et al. (2012), Shakshuki et al. (2015), Zhan et al. (2016), Suh et al. (2012), Price et al. (2015), Abramiuc et al. (2015), Lam et al. (2015), Dhulipala et al. (2016), Sola-Valls et al. (2015), Casacci et al. (2015), Karakostas et al. (2015), Almagooshi et al. (2015), Naslund et al. (2015), Schneider and Biglan (2017), Giggins et al. (2017), Block et al. (2016), Prabhakar and Rajaguru (2017) and FaurholtJepsen et al. (2014)

Rodbard (2016), El-Gayar et al. (2013), Vashist (2012), Hood et al. (2016), Szydlo and Koneiczny (2015), Kozlovszky et al. (2015), Rasmussen et al. (2015), Fioravanti et al. (2015), Suh et al. (2012), O'Grady et al. (2012), Ly et al. (2014), Askew et al. (2012) and Hazenberg et al. (2012)

Aldaz et al. (2015), Guo et al. (2012), Ferreira et al. (2015), Mobasheri et al. (2015), Bauer and Mendes (2015), Rotariu et al. (2015), Khattak et al. (2014), Gonzalez et al. (2015), Patel et al. (2012), Hegde et al. (2013), Benelli et al. (2012), Zhang et al. (2015), Magno et al. (2013), Sardini et al. (2015), Sorwar and Hasan (2012), Serhani et al. (2016), Al-Naji et al. (2017) and Wang et al. (2015a, b) 
category, two further classifications present contact-based and contactless efforts.

Summary of reference articles classified by the target diseases are given in Table 1.

\section{Remote patient monitoring systems by diseases}

\subsection{Heart and blood related diseases monitoring systems}

Heart related monitoring systems are the most common type of monitoring systems. The reason for this could be that the vital signs associated with heart could relate to many illnesses at the same time revealing many hidden illnesses. Cardiac arrhythmia, chronic heart failure, strokes, blood clots and high blood pressure are some of the most common illnesses in this subcategory. The possibility to measure ECG, heart rate, respiration rate, blood pressure, oxygen volume in blood and detection of arrhythmias have been discussed in literature. Various technologies such as actual ECG monitors or textile-based wearable systems are used to get these data. Although these essential data can be gathered, there is much room for improving the accuracy of these systems.

Various applications that use smartphone in various aspects of cardiology such as remotely monitoring patients and user guidance applications for prevention of cardiac diseases are analysed in the review paper (Nguyen and Silva 2016).

Challenges This type of monitoring systems is the most common as heart associated illnesses are the prominent cause of mortality in the world. Also, vital signs monitoring system usually gives the overall results together with heart and respiration related measurements.

One of the main challenges in this area is to obtain a clean signal from a patient's body. Contact-based methods usually use traditional methods like ECG and while photoplethysmographic methods use light incident on small veins closer to the skin surface and these have proven useful. Detecting breathing abnormalities and respiratory system problems challenge the monitoring systems because these systems include mainly breathing sound detection. As the upper body produces many sounds, differentiating these is difficult.

We start the discussion with contact-based methods and then move on to contactless systems.

Contact-based methods A 'telemonitoring system' uses hardware and software devices (Szydlo and Koneiczny 2015) to monitor various heart-related illnesses. Software applications run on android platform. ECG and heart rate variability detection method based on Autonomous Nervous Systems is given in Lanata et al. (2015). In Kozlovszky et al. (2015) cardiovascular diseases are monitored using off-the-shelf sensor sets. An analysis of effectiveness of cardiac implantable electronic devices is given in Ricci et al. (2013). ECG and Blood pressure monitoring system given in Ramesh et al. (2012) has an algorithm based on five states where the mobile device can be of different states depending on its charge level. Thus this has energy optimization feature where even the data storage will be done while saving energy. A non-invasive body temperature monitoring system is presented in Ling et al. (2015). Another system (Kumar and Kotnana 2012) measures a person's heart rate, ECG, pulse oximetry, $\mathrm{pH}$ level of blood and temperature using a series of sensors. Ferreira et al. (2015) explain a system for bedridden people under the concept of ambient assisted living. This system can measure ECG, body temperature, air flow in lungs, oxygen saturation levels, and galvanic skin response. Although Sannino et al. (2015) is a full fall detection system, to aid its decisions, it gathers accelerometer readings and vital signs such as ECG, SpO2, temperature, heart rate (HR), heart rate variability (HRV). Otoom et al. (2015) discuss about developing an application for real-time diagnosis and monitoring of patients with coronary artery disease and heart diseases. Their classification process is $85 \%$ correct while detection works $100 \%$. It works in three modes (rest, sports and drive), and has a ten-fold algorithm based on support vector machines (SVM) to aid its decision making.

Using a telemedicine scenario, where two paramedics from inside of an ambulance communicate to a 'tele-EMS physician' located in 'teleconsultation centre' in a remote location, it has been shown that simple web applications and simple device interfaces are better than using specialized network protocols and off-the-shelf devices in emergency telemedicine systems, since the former does not put any constraints on users as well as developers (Thelen et al. 2015). Apart from being a monitoring system for joint angle and hydrotherapy, the system in Alves et al. (2015) has the capability to obtain vital signs such as oxygen saturation and pulse. The paradigm of using smartphone as a 'hub + sensor + processor' manner is introduced and developing personal area network upper layers architecture for Android phone has been presented (Bisio et al. 2015a, b). A comparison between five remote health monitoring applications (Papon et al. 2015) shows promising results for some of the with-contact methods. The incorrect results for the contactless method have been concluded as problems due to ambient lighting. Disadvantages of lack of deterioration monitoring have shown to be minimised with Patient Guard 300 (PG300) body worn apparatus which measures ECG, heart rate, respiration information and temperature (Donnelly et al. 2015). Mishra and Agrawal (2015) presents obtaining physiological data related to pulmonary artery pressure (PAP) and ECG and encoding the data as a text message. It has been 
discussed that a dedicated channel for medical data with 5 th Generation (5G) mobile systems can further improve $24 \times 7$ monitoring with this system.

System architecture for sensing cardiac data for a wheelchair user (Pinheiro et al. 2013), Photoplethysmography (PPG) imaging using Oxicam (Karlen et al. 2014) and false alarm reduction system (Tanantong et al. 2015) are some recent findings. Also usage of piezoelectric sensors for heart-related medical readings and seismocardiogram (Bifulco et al. 2014) is found and similarly HeartCycle (Luprano et al. 2013) uses textile-based sensors. In Ganesan et al. (2015), Bayesian algorithm is used to find heart rate abnormalities. Enhanced Fourier Interpolation method is used in the capacitive electrocardiography and heart rate monitoring system (Thakur and Anoop 2015). This system has been verified with different cloth thicknesses.

Table 2 summarises the technologies discussed in this section.

Contactless methods Another review on camera based methods has been published very recently (Hassan et al. 2017). Cuffless blood pressure monitoring systems have been reviewed by Sharma et al. (2017).

A system for remotely assessing heart rate by skin colour processing is given in Bogdan et al. (2015). It uses principal component analysis and empirical mode decomposition. Heart rate measured using a web-camera has been proven accurate by conventional ECG readings (Monkaresi et al. 2014). Respiration rate has been similarly found using a web camera (Lukáč et al. 2014). Kinect version 2 camera too has been found to be accurate proven by a study to measure respiration in neonates. Chandrasekar et al. (2012) show the usage of optical proximity sensors (OPS) in photoplethysmography to obtain physiological signals. A blood pulse measurement system using linearly polarized light is shown in Mishra et al. (2015).

Use of pupillary fluctuations is another way to measure heart rate variability (Parnandi and Gutierrez-Osuna 2013). Segmentation of the boundary of the eye pupil and a remote eye tracker are essential here, in order to see the pupil's diameter changes that occur with heart beat.

A web-based interface for optical coherence tomography image processing for diagnosis of diseases related to retina is presented in González-López et al. (2015). Other facial signs can also be used to derive many data associated with cardio-metabolic events. These require camera, multispectral imaging system and 3D optical sensors according to Colantonio et al. (2015).

Use of dual-wavelength imaging system to find blood oxygen saturation is shown in Shao et al. (2016). Planar and avalanche photodiodes against IR and visible light wavelengths for the purpose of usage as non-invasive cardiovascular monitoring are compared in paper (Pereira et al. 2013). The concept of 'pulse harmonic strength' is developed in contactless research (Couderc et al. 2014) which uses facial video signal to detect atrial fibrillation. In Sugita et al. (2015), pulse transit time has been shown as a measure to obtain blood pressure (BP) information from video images. A system that performs pulse rate variability analysis using videos is presented in Rodríguez and Castro (2015). In paper (Alzahrani and Whitehead 2015), a similar approach is discussed but this system has Eulerian video magnification and face tracking. The research described in Janssen et al. (2016) measures thoracic impedance plethysmography using Phillips Intellivue MP50 and CCD-RGB camera. This system has automatic region-of-interest (ROI) detection. It uses feature extraction methods and singular value decomposition (SVD) or principal component analysis (PCA) for the processing.

A Kinect camera has been used in Bernacchia et al. (2014) for measurement of heart rate and respiration rate. A non-contact video based method is used to obtain vital signs, heart rate and respiration rate of patients undergoing haemodialysis (Tarassenko et al. 2014). This system uses a high definition camera.

Elfaramawy et al. (2017) discuss a system that has both contact-based and noncontact mechanisms. The wireless system monitors respiration while a wearable system detects a patient coughing. Inertial measurement units are used to measure thoracic and cavity motions for respiration monitoring while a MEMS microphone is used to record coughing and air sounds from chest. While this system is claimed to be a contactless system for respiration monitoring it is not fully non-contact as the IMU units are attached to the chest. Only the readings are sent wirelessly. However, as the IMU nodes are small low-weight nodes the person is free for activities therefore this is an advantage. The nodes also require low power $(3.7 \mathrm{~V})$ and the system can detect talking and differentiate coughing. This is a very promising system which uses zero crossing rate (ZCR) and filters (FIR and Savitzky-Golay smoothing filter) for the processing. It also suggests more complex algorithms for improvement. Table 3 summarise these contactless systems.

\subsection{Fall detection and mobility related disease monitoring systems}

Fall detection systems are an essential type of system especially for elderly persons monitoring. These systems too appear as contact-based and contactless as these systems target mainly elderly community and post-accident recovery patients. Some reviews done recently summarize the technology advancement so far.

Challenges Main challenge fall detection systems face is differentiating a fall from activities of daily living. Many systems include accelerometers which give data related to movements and the algorithms must be robustly designed 


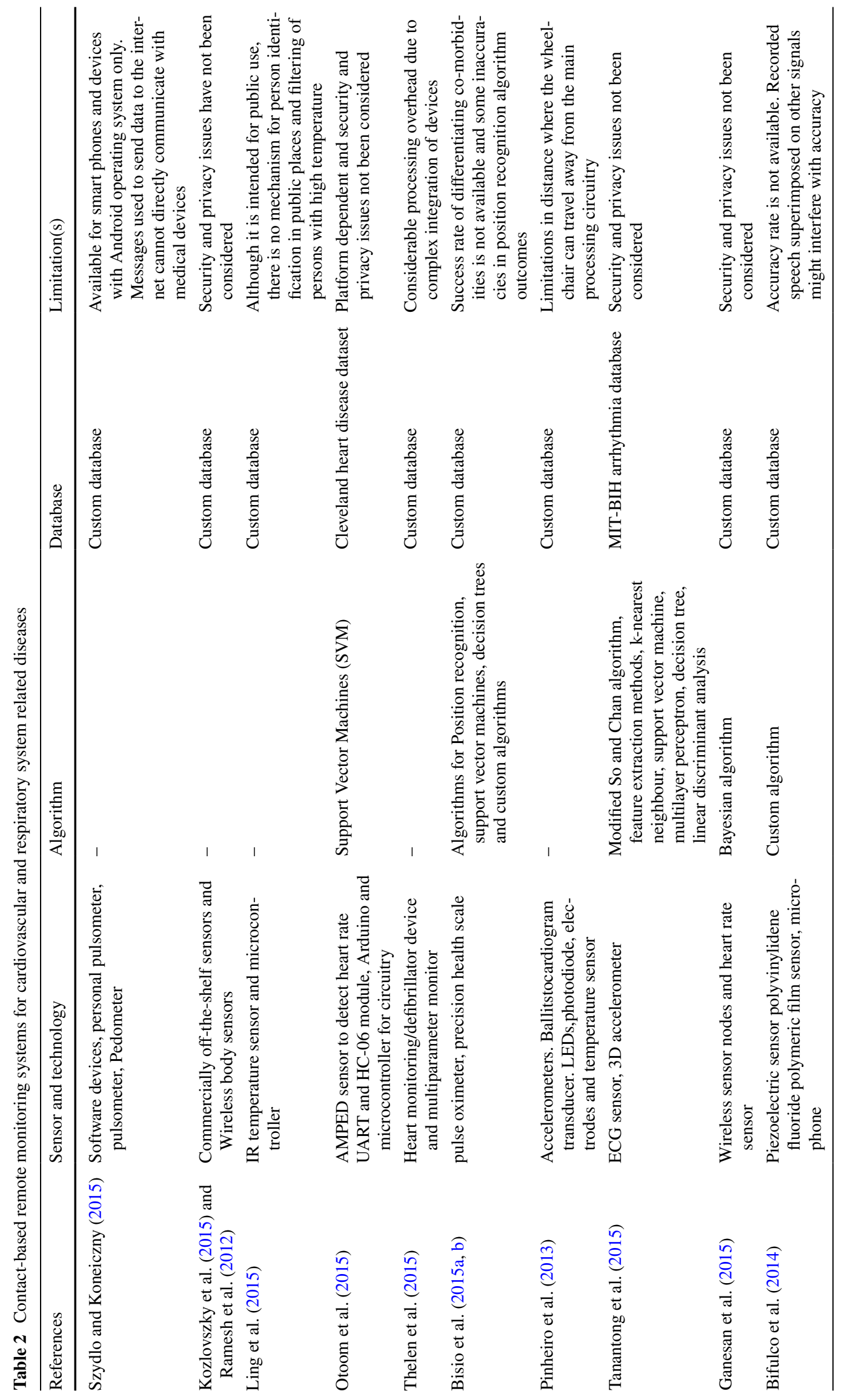


so that it correctly identifies falls from a quick movement like bending down to pick something from the floor or quickly sitting down. Generally false alarms are common in this kind of systems. The next challenge is the lack of real fall data for improving research. As falls are rare, diverse and infrequent incidents, recording or obtaining these data are hard and at present, sufficient data does not exist. Although databases of fall data where falls were performed by young and healthy subjects exist, these may not represent an actual fall an elderly person undergoes in real life. Also, collecting fall data under controlled environments by healthy and young adults too can be harmful therefore researchers also can find ethics clearance from academic institutions to be a difficult procedure.

Out of the reviews on fall detection systems, we have chosen a few papers that are very important. Hegde et al. (2013) discuss about recent technical advancements in fall detection systems. Meanwhile, Igual et al. (2013) compare many models, classifiers and algorithms and novel techniques for detecting fall such as monitoring inactivity level after a fall.

Mubashir et al. (2013) concentrate on fall detection systems in their survey paper. The fall detection systems are classified into three types; wearable device based, ambience device based and vision based. In this survey, systems including posture based devices, motion based devices, vibrations and presence detection methods, use of shape change, inactivity detection and three-dimensional (3D) head motion detection methods are discussed. The systems discussed here consist of one or more of the following; different types of accelerometry, vital physiological data collection, audio, video and vibrational data collection and single or multi-camera data acquisition. Support Vector Machines, Gaussian Models, Markov Models and Neural networks with Fuzzy Logic have been shown to be the popular classifiers used in this kind of RPM systems.

Another very recent review on fall detection techniques further narrows down the conversation to 'a data availability perspective' (Khan and Hoey 2017). They argue that sufficient data on falls may not be available during training classifiers of the systems that use supervised machine learning methods. They suggest that semi-supervised machine learning methods, anomaly detection, varied sampling methods (under/over-sampling), cost sensitive classification and one class classification methods may be more efficient than traditional heuristic supervised learning methods. They also provide a taxonomy for this kind of studies based on the availability/lack of data which is independent of the type of data acquisition used.

Contact-based methods Some of the main problems the elderly community has today are the number of falls and issues in mobility. Also, there can be many young patients who have the same issues due to malnutrition, conditions like polio or as the after effects of accidents. Therefore, fall detection and mobility related systems play a major role in remote monitoring of this category of patients. The research in recent literature show very successful systems. One common issue that needs more attention is detecting a fall from a sudden change of posture because most systems are prone to false positives. Also system adaptation to the person must be further investigated. This section gives an overview of fall detection and mobility related diseases monitoring systems and their pros and cons.

A new rule based (IF-THEN rules) system for fall detection with an embedded sensor which has the capability to detect fall without connecting to a database is given in Sannino et al. (2015). The novelty of this application is twofold; that the rules are extracted automatically and the same application layer used for extracting rules does the processing tasks. A system that is useful for assessing joint angle and other vital signs to therapists with the capability to use in hydrotherapy is given in Alves et al. (2015). Wang et al. (2013) discuss about a fall detection and activity monitoring system based on android Smartphone. (Gibson et al. 2016) present a multiple comparator and classifier framework for fall detection.

A fall detection system with wireless motes and with localization capability is described in Paoli et al. (2012). A smart sensor with an accelerometer is used in NaranjoHernandez et al. (2012) together with a processing device. A similar effort is given in Bai et al. (2012). E-FallD (Cao et al. 2012) is also a fall detection system using threshold algorithms. In Lanata et al. (2015), movement is also measured and monitored in addition to its main application of mental health monitoring.

Contactless methods The papers Yu et al. (2012) and Mastorakis and Makris (2014) are on a camera-based fall detection system. Additionally, in Mastorakis and Makris (2014), IR capabilities available in Kinect sensor have been used. This too uses post-fall inactivity detection to achieve accurate fall detection. A directed acyclic graph support vector machine has been used in Yu et al. (2012), in addition to rule-based algorithms and background subtraction algorithms.

Greene et al. (2016) present an Internet of Things-based fall detection system. This system uses a fall detector; an amazon echo device, a speaker and a webcam (Logitech c920) for this function. These devices are interconnected by a hub which consists of a raspberry pi model $\mathrm{B}+$. The fall detector is made from Tiva C Launchpad, a WIFI expansion board and an accelerometer. When a fall is detected it is then communicated to the hub and then speakers are used to interact with the person. Amazon echo device has voice recognition capability and get the confirmation from the user about the fall. This system effectively reduces false positive rates as it gets the fall confirmed and whether to 


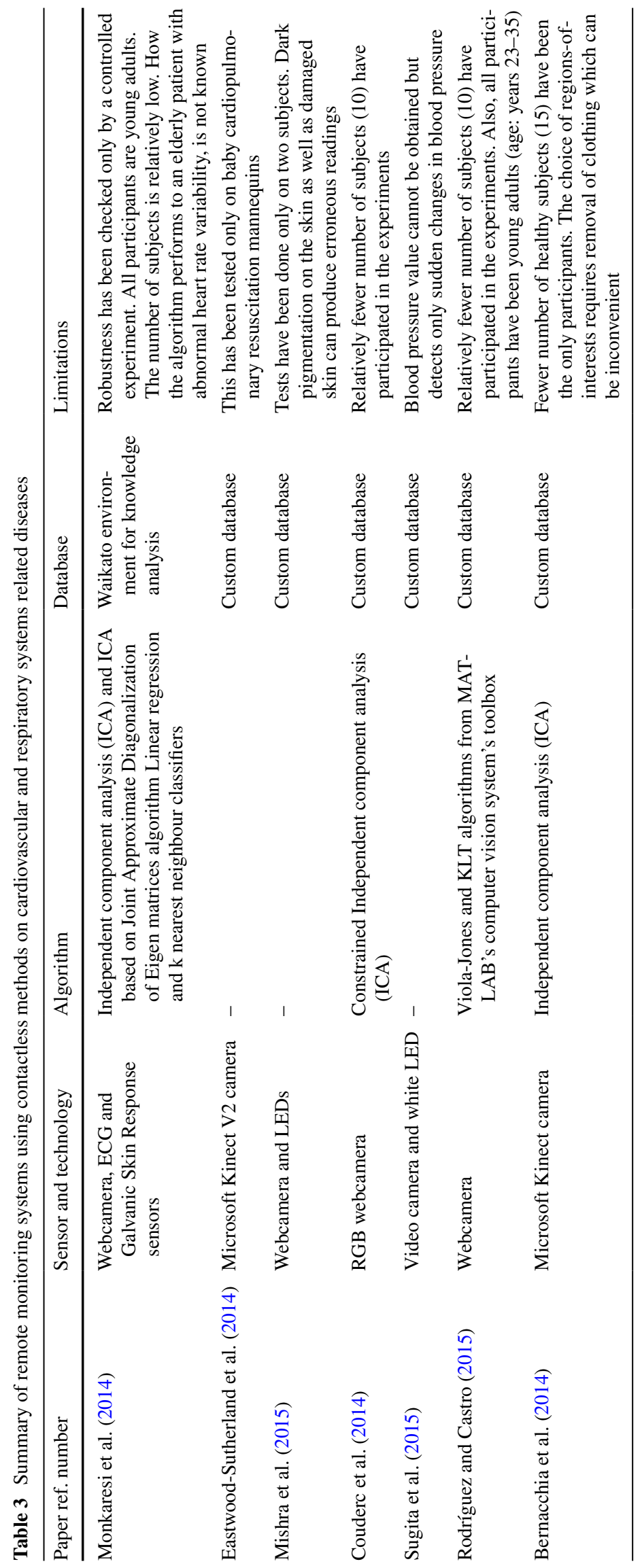




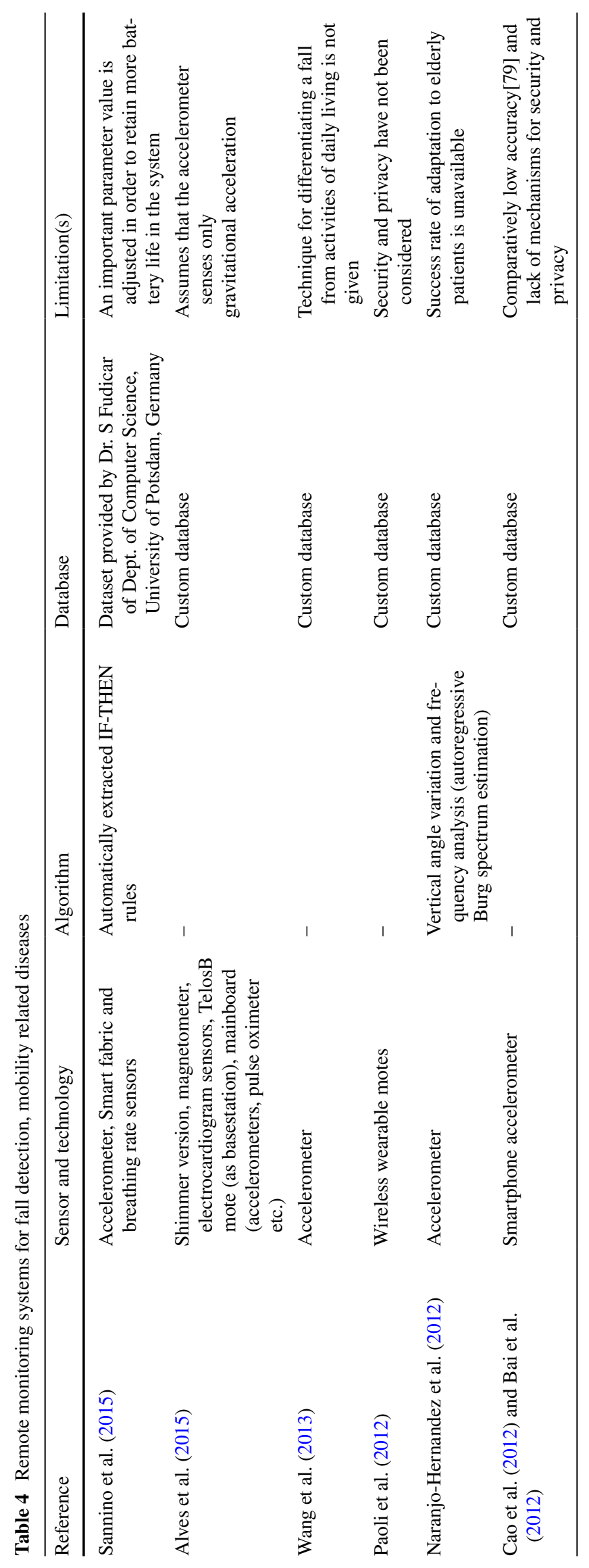


communicate this fall to the corresponding parties and get help is based on the actual confirmation by the user.

Cippitelli et al. (2017) discuss contactless fall detection monitoring in their review paper. This paper discusses both radar based and RGB and Depth data based methods for fall detection.

Summary of the systems given in this section is given in Table 4.

\subsection{Monitoring system for brain, neurological system related diseases and mental health}

This section focuses on neurological system related diseases including those caused by an abnormality of or an injury to brain and neurological system. Mental health is discussed together with this as these two branches of diseases tend to go hand in hand in most cases.

Challenges The designing of monitoring systems for these diseases challenge from the fact that some diseases may have a lot of related diseases that demands for systems with vital signs, autonomous nervous system responses and psychiatric responses. So the systems can be more complex than other monitoring systems.

In the survey paper (Wac and Tsiourti 2014), monitoring of emotion changes using autonomic nervous systems has been presented. The focus is on ambulatory assessment. Paper (Naslund et al. 2015) is a review of technologies for monitoring patients with serious mental illnesses. Analysis of the possibility of using telemedicine to monitor the progression of multiple sclerosis patients is given in Sola-Valls et al. (2015).

A recent survey paper discusses the extent of remote patient monitoring and telemedicine has reached its use in neurological disorder detection at present (Schneider and Biglan 2017). The paper's main focus is on Parkinson's disease and it shows that the patients' acceptance of use of telemedicine is increasing with a recorded $100 \%$ acceptance rate in a study carried out in 2014. This needs to be further investigated with more subjects (this study has 44 subjects), but it is a promising result. Additionally, the feasibility of using remote monitoring and telemedicine in other neurological disorders such as epilepsy, dementia, depression, consistent non-acute headaches and multiple sclerosis are briefly discussed. Some major issues of telemedicine are also pointed out; access (from rural areas in most parts of the world), usability (the patients are more likely to be the elderly with less experience in technology and weaker physical and cognitive ability to use technology) and reimbursement of costs (as only certain telemedicine providers are licensed with standard insurance companies). Also, authors highlight that there is very little evidence to prove that care through telemedicine and care in traditional in-person clinic is roughly the same.
Giggins et al. (2017) review the advancement and usage of novel body-worn devices for activity monitoring of patients with neurological disorders. The paper analyses studies that used subjects with various neurological illnesses such as Parkinson's disease, types of cerebral palsy, Rett syndrome, multiple sclerosis, muscular dystrophy, paraplegic participants and patients suffering with spinal cord injury, traumatic brain injury, post-stroke and chronic stroke patients and found out that accelerometer as the best method to monitor activities of these patients.

A larger survey on existing technique critically analyses various activity monitoring techniques of patients with Multiple sclerosis, Parkinson's Disease, dementia, traumatic brain injury and ataxia (Block et al. 2016). They summarize how remote monitoring can help these patients as per the disease. They show that remote monitoring of MS shows the extent of disability and the low quality of life which is a result of decreased ambulatory activity. Goal-directed interventions and recovery together with rehabilitation is aided by remote monitoring of stroke patients. Remote monitoring can help fall prediction of Parkinson's disease patients while it can detect wandering of patients with dementia.

Lanata et al. (2015) gives a system for monitoring mental health patients suffering from mental bipolarity. Based on textile-based autonomic nervous sensor system, this system gathers speech during tasks and features questionnaire system, sleep activity and medication intake monitoring capabilities. A study cited in Nadeem et al. (2015) mentions EEG signal sensing using block sparse Bayesian learning. Karan et al. (2012) present an artificial neural networks based system for monitoring diabetic patients. HopkinsPD, a system to monitor patients suffering from Parkinson's disease has active and passive monitoring capabilities (Zhan et al. 2016).

A cognitive fatigue monitoring system for people with acquired brain injury is given in Price et al. (2015). Cognitive tests from Wechsler Adult Intelligence Scale (WAIS), Psychomotor Vigilance Test and Serial Addition Subtraction Tasks are used for monitoring. The system uses only a smartphone for performing these.

A recent study has developed a remote patient monitoring system for epilepsy classification (Prabhakar and Rajaguru 2017). The main purpose of the paper is to use dimensionality reduction on collected EEG data. It is done by Fuzzy Mutual Information (FMI). A new approach of Singular Value Decomposition Based Partial Transmit Scheme (SVDPTS) is introduced for transporting the data and Gaussian Kernel Based Support Vector Machine (G-SVM) is used for classification. They report their accuracy to be $95.38 \%$ with a time delay of only $2.19 \mathrm{~s}$.

Remote patient monitoring in psychiatry for better biomarker data acquisition and thereby improve psychiatric research on utilizing mHealth for psychiatry is presented in Adams et al. (2017). The paper shows the usage 
of body-worn or contact-based biosensors for this kind of study. Additionally in most studies, a self-reported form explaining the patient's mood and stress levels are also collected. ECG sensors, Galvanic Skin Response (GSR) sensors, multi-sensor units that look like wrist watches or wrist cuff, Salivary Alpha Amylase biosensors are used. Also the authors consider that vision-based methods to extract vital signs such as heart rate variability, respiration rate too can be used for psychiatric patient monitoring as these physiological data can indicate a person's stress level. Also this kind of mobile biosensing can be used for those who are addicted to smoking, alcohol and illicit substance use. The research reports several studies that use non-invasive methods such as breath carbon monoxide monitoring, chest expansion and hand movement monitoring (for smoking), alcohol breathalysers and biomarkers in perspiration (for alcohol use) and electrodermal activity and skin temperature (for illicit drugs). This can also help people with mood disorders, attention-deficit hyperactivity disorder (ADHD) and autism.

Faurholt-Jepsen et al. (2014) have studied the correlation between smartphone application data with bipolar disorder symptoms. A software called 'MONARCA' has been used with 17 patients for three months. The software has a set of questions to give short answers on depression symptoms (i.e. mood, sleep, alcohol consumption etc.), while it also monitors number of calls, texts, speech data and activity level using an accelerometer. This study shows that there is a significant correlation between smartphone data and selfrated depression symptoms rated on Hamilton Depression Rating Scale-17 (HDRS17) with p-values below 0.05.

Contactless methods Smart Mind (Lam et al. 2015) is a remote monitoring and an activity tracking system for patients with Alzheimer's disease. It uses a Kinect for activity tracking and has proven good accuracy rates for overall monitoring with the use of support vector machines and naïve Bayes algorithms. Random forests algorithms are also used, but the accuracy rate is low compared to other two algorithms. Similarly in Casacci et al. (2015) a project called ALTRUISM project is given which uses a Kinect (with gesture recognition), a set-top box, a TV and a wearable system for Alzheimer's monitoring. The development of a prototype to measure the parameters of such patients are given in Almagooshi et al. (2015). The paper Dhulipala et al. (2016) is a paper on stress level monitoring depending on blood pressure measurements. Heuristic algorithms are used here. A system to monitor patients with dementia is given in Karakostas et al. (2015). It uses multiple sensors; Asus Xtion Pro camera, Plugwise sensors and Wireless Sensor Tag System and a wristwatch 5DTI-2 (Philips). Complex Activity Recognition (CAR) and Offline Speech Analysis (OSA) algorithms are used.

Table 5 Brain, neurological system related diseases and mental health monitoring systems

\begin{tabular}{|c|c|c|c|c|}
\hline References & Sensor and technology & Algorithm & Database & Limitation(s) \\
\hline Kozlovszky et al. (2015) & $\begin{array}{l}\text { Textile based autonomic } \\
\text { nervous system }\end{array}$ & - & Custom database & No alarm system is available \\
\hline Zhan et al. (2016) & $\begin{array}{l}\text { Smartphone based tests for } \\
\text { gait, reaction time, balance }\end{array}$ & Random forest classifier & $\begin{array}{l}\text { Database from } \\
\text { Parkinson's Voice } \\
\text { Initiative }\end{array}$ & Only $71 \%$ accuracy is obtained \\
\hline Suh et al. (2012) & $\begin{array}{l}\text { Diabetic patient monitoring } \\
\text { system }\end{array}$ & $\begin{array}{l}\text { Data association rule learning } \\
\text { algorithms }\end{array}$ & Custom database & $\begin{array}{l}\text { Lack of a mechanism for ensur- } \\
\text { ing security and privacy }\end{array}$ \\
\hline Price et al. (2015) & $\begin{array}{l}\text { Smartphone based cognitive } \\
\text { fatigue assessment and } \\
\text { monitoring }\end{array}$ & - & Custom database & $\begin{array}{l}\text { Lack of immediate actions for } \\
\text { serious fatigue levels }\end{array}$ \\
\hline Abramiuc et al. (2015) & High Definition Camera & - & Custom database & $\begin{array}{l}\text { Lack of emergency assistance, } \\
\text { post-processing actions } \\
\text { (medication, physiotherapy or } \\
\text { rehabilitation) and mecha- } \\
\text { nisms for transferring data to } \\
\text { another location (to doctor / } \\
\text { hospital) }\end{array}$ \\
\hline Dhulipala et al. (2016) & Digital sphygmomanometer & - & Custom database & Accuracy rate is not available \\
\hline Casacci et al. (2015) & $\begin{array}{l}\text { Set-top box, Kinect camera, } \\
\text { t-shirt with embedded } \\
\text { sensors }\end{array}$ & - & Custom database & $\begin{array}{l}\text { Lack of a mechanism for ensur- } \\
\text { ing security and privacy }\end{array}$ \\
\hline Karakostas et al. (2015) & $\begin{array}{l}\text { Asus Xtion Pro camera, Plug- } \\
\text { wise sensors and Wireless } \\
\text { Sensor Tag System and a } \\
\text { wristwatch }\end{array}$ & $\begin{array}{l}\text { Complex Activity Recogni- } \\
\text { tion (CAR) and Offline } \\
\text { Speech Analysis (OSA) } \\
\text { algorithms }\end{array}$ & Custom database & $\begin{array}{l}\text { Security and privacy issues not } \\
\text { been considered }\end{array}$ \\
\hline
\end{tabular}


A camera based method is implemented in Abramiuc et al. (2015) to monitor patients with neurodegenerative diseases. A high-definition HC-V720 camera is used to obtain videos. In this system, after detecting the human, armswings and step-length are used as parameters to monitor. From these parameters, a person's status of health (in the point of view of neurodegenerative diseases) can be analysed (see Table 5).

\subsection{Diabetes monitoring systems}

A recent study compares continuous glucose monitoring methods and traditional clinic based glucose monitoring by a professional and assess the pros and cons of the two types in terms of usage, accuracy, acceptability (Rodbard 2016).

El-Gayar et al. (2013) review commercial applications between January 1995 and August 2012 which are available on the Apple App Store. The chosen applications are on self-management tasks to reduce diabetes and manage life with it. The review suggests that improvements should be made in areas such as usability, integration between patients and electronic health records, and on giving personalized feedback.

Vashist (2012) reviews many non-invasive glucose monitoring techniques available today. They include electrode based, light and sound based methods. The review clearly lists the advantages and disadvantages of each of these methods and analyses some commercially available glucose monitoring devices. Tremendous improvements are required in these devices in terms of accuracy, precision, cost and quick response.

A very recent review presents an analysis on the past reviews on diabetes self-management mobile applications (Hood et al. 2016).

Contact-based systems for diabetes EEG and glucose monitoring capability is discussed in Szydlo and Koneiczny (2015). The remote monitoring system given in Kozlovszky et al. (2015) uses off-the-self sensors for diabetes sensing. In Rasmussen et al. (2015) a qualitative analysis has been done on implementing a remote monitoring system for diabetic foot ulcer patients. An automatic feedback message generation in diabetes management system (Fioravanti et al. 2015) has the capability to record food intake, blood pressure measurement, blood glucose level, weight, drug intake and physical activity and features functionalities for chatting (doctor and patient) and a message repository which has messages to be sent to the patient as necessary.

A system for diabetic patient monitoring which is verified on Weight and Activity with Blood Pressure and Other Vital Signs (WANDA) project is given in Suh et al. (2012).

A research that uses a portable glucose control system for overnight glucose monitoring and control is presented in O'Grady et al. (2012). It uses a Medtronic Portable
Glucose Control System (PGCS) which includes glucose sensors and a Medtronic Paradigm Veo insulin pump. The system's control algorithm communicates using Black-Berry Storm smartphone platform and a Bluetooth radio frequency translator. After the communication between the sensors and insulin pump is performed the required amount of insulin is administered to the patient. This system targets patients with diabetes 1 . Also this system uses a separate venous plasma glucose monitoring sensor and when the glucose amount is at dangerous levels in two consecutive readings, the sensing will be stopped and insulin will be given. These two experiments were done separately on patients and the feedback mechanism of the first system and its closed-loop nature of operation has shown better results and better maintenance (i.e. for longer durations) of patients' blood glucose than the second open-loop method.

A similar closed loop system is described in Ly et al. (2014). This has used different sensors (Dexcom G4 Platinum glucose sensor), a separate mobile phone (Sony Ericsson or Xperia); then the data is transmitted to a PC that uses a web based program which can be accessed by medical professionals remotely to do remote monitoring.

Contactless systems for diabetes Askew et al. (2012) discusses contactless methods on diabetic retinopathy screening. Photographs of retina are taken from time to time and patients are treated at a primary care unit while ongoing care is given via tele-ophthalmologist consultation using means of tele-conferencing.

A relatively early study (Hazenberg et al. 2012) has assessed the feasibility of using a photographic foot imaging device to monitor diabetic foot ulcers. Then the health related quality of life was analysed and it seemed to improve the health related quality of life of patients.

\subsection{Other related applications}

This section discusses a few other healthcare related applications.

SnapCap wound management system features a Google Glass application and an android application with voice based commands (Aldaz et al. 2015). This system can operate according to voice commands and gestures of the operator such as tilting or blinking eyes.

Improvement of network traffic using appropriate software development methods have been presented in Ling et al. (2015). Ambient assisted living projects feature room luminosity sensors and mechanisms to measure room temperature and air quality and composition (Ferreira et al. 2015). The smartphone and tablet usage in surgical scenarios are looked into in Mobasheri et al. (2015). Cardiac, ear-nosethroat (ENT) and general surgeries, image analysis of joints have been discussed. A weblab (web browser interface) of 
a baby incubator monitoring system is given in Bauer and Mendes (2015). Big data capabilities in healthcare are discussed in Wang et al. (2015b). Smartphone assisted technology for eye care have been shown to be promising (Lakshminarayanan et al. 2015). Bioimpedance that can be used for composition analysis, respiration analysis, melanoma and skin related diseases have been shown in an application using wireless sensor network (Rotariu et al. 2015). In Khattak et al. (2014), a constrained application protocol based low power personal area network used as a healthcare sensor network is presented.

Another discussed application is pre-hospital assistance; especially when a victim of an accident or some kind of injury is being transported to a hospital in an ambulance (Gonzalez et al. 2015). Patel et al. (2012) discusses about wearable sensors and systems in rehabilitation applications with an emphasis on development of microelectromechanical systems (MEMS) and system-on-chip implementations. A thin copper wire running on a $t$-shirt and a piezoelectric actuator are used in a posture monitoring system used during rehabilitation (Sardini et al. 2015). Benelli et al. (2012) present an outcome of a research collaboration, a system to measure many conditions (blood pressure, body weight, glycaemia, ECG, spirometry) and this shows the usage of a television as the output interface. Similarly, the system in Sorwar and Hasan (2012) also uses television for a multiparameter monitoring system. A remote rehabilitation system shows the usage of two devices each at the therapist (master device) and patient (slave exoskeleton device) working synchronously to aid with rehabilitation activities (Zhang et al. 2015). E-Ambulance (Shakshuki et al. 2015) is a remote monitoring system of patients while they are being transported to a hospital in an ambulance with the ability to monitor vital signs. A Smart Rehabilitation Garment (SRG) has been developed in Wang et al. (2015a) for posture correction. A low-power architecture for wireless on-body sensor network is presented in Magno et al. (2013). Smart Mobile End To End Monitoring (SME2EM) architecture for life-long disease monitoring is a set of web services utilizing Data as a Service (DaaS) concept and a custom algorithm which combines filter and wrapper selection algorithms (Serhani et al. 2016).

Camera based children monitoring in hospital environments is given in Al-Naji et al. (2017). It measures respiration rates and detects apnoea based on Kinect camera.

\section{Future research directions and trends}

Although the technology has advanced over the years, there are some significant lacks in the research. Some of the main issues that have not been addressed to the required level 
are the security and privacy issues. All of the above literature shows good technology usage, but only a few discusses about their systems' capabilities for ensuring privacy and security. For example, in Sawand et al. (2015) there is a good discussion about most relevant security and privacy issues in wireless sensor networks, but jamming attacks where one or few of the sensor nodes are compromised due to an attack is highlighted as an area which needs more investigation.

It is quite clear that most systems are confined to a specific operating system (Szydlo and Koneiczny 2015). This makes it difficult to combine devices with other operating systems and this is a major limitation in most researches.

Issues with usage are unique for every system. The general procedures of obtaining physiological data can be common to many systems. System adaptability to the person is very important. For instance, the fall detection systems suffer from the issues of adapting to each and every patient because every individual has unique gait values which make it difficult for systems to be designed under a common set of design parameters. Therefore this issue must be resolved in designing fall detection systems. The other issue with usage is comfort of the patient. The comfort of a patient can directly or indirectly influence physiological readings to a certain extent. The choice of sensors is therefore imperative. Some systems have very low comfort to the patient (Ferreira et al. 2015).

Contactless image-based methods have a lot of developments to be made. For example, motion artefact removal has not been fully solved. In this, patient motion as well as camera's motion must be addressed. Also lack of a dataset to be used in research also is mentioned in McDuff et al. (2015). The world moves fast towards contactless methods, newer technology needs to be found for contactless methods. This paper only discussed about image-based methods. However there exists radar/microwave based methods. A combination of the two technologies could be a new path for research.

Although researchers, together with the medical professionals may like to move towards contactless methods, it might not be feasible or advisable at this stage of research as there are still a few significant problems to be solved in contactless monitoring. Therefore, before moving to completely contactless systems, some combination of both types can be more reliable. From this review, we can summarise a few approaches that can be useful for systems with higher accuracy, precision, dependability and usability. These are given in Table 6.

Overall, more studies need to be done to see the acceptance of these technology based methods with the medical community and patients. Although some trial studies have been done, error correction methods in the technology have not been able to win the medical professionals' complete trust.

\section{Conclusion}

This paper has reviewed remote patient monitoring systems in the current literature. Heart and blood related systems, fall detection systems, brain and nervous system related systems, diabetics and mental health research have been discussed. Most recent development in contactless camera-based methods has also been discussed here. Based on many categories the existing research has been presented. The review shows that this emerging field of technology is making substantial impact on the society as well as the research community. As the technology advances, the outcomes are also increased. The paper concludes with some suggestions for future research.

Acknowledgements This project is funded by "SmartLink: SouthEast-West Mobility for Advanced Research, Learning, Innovation, Network and Knowledge" project which is funded under Erasmus Mundus Action 2 (EMA2) - Strand I - Call for Proposals EACEA/18/13.

\section{Compliance with ethical standards}

Conflict of interest The authors declare that they have no conflict of interests.

Open Access This article is distributed under the terms of the Creative Commons Attribution 4.0 International License (http://creativecommons.org/licenses/by/4.0/), which permits unrestricted use, distribution, and reproduction in any medium, provided you give appropriate credit to the original author(s) and the source, provide a link to the Creative Commons license, and indicate if changes were made.

\section{References}

Abramiuc B et al (2015) Home video monitoring system for neurodegenerative diseases based on commercial HD cameras. In: Consumer electronics-Berlin (ICCE-Berlin), 2015 IEEE 5th international conference on, pp 489-492

Adams ZW, McClure EA, Gray KM, Danielson CK, Treiber FA, Ruggiero KJ (2017) Mobile devices for the remote acquisition of physiological and behavioral biomarkers in psychiatric clinical research. J Psychiatr Res 85:1-14. doi:10.1016/j. jpsychires.2016.10.019

Aldaz G et al (2015) Hands-free image capture, data tagging and transfer using google glass: a pilot study for improved wound care management. PLoS One 10(4): 1-21. doi:10.1371\%2Fjournal. pone. 0121179

Almagooshi S et al (2015) An assisted living home for alzheimer's patient in saudi arabia, a prototype. In: HCI international 2015-Posters' extended abstracts: international conference, HCI International 2015, Los Angeles, CA, USA, August 2-7, 2015. Proceedings, Part II, ed. Constantine Stephanidis. Cham: Springer International Publishing, 375-80. doi:10.1007/978-3-319-21383-5_63

Al-Naji A, Gibson K, Lee SH, Chahl J (2017) Real time apnoea monitoring of children using the microsoft kinect sensor: a pilot study. Sensors 17(2):286

Alves RCA et al (2015) Assisting physical (hydro)therapy with wireless sensors networks. IEEE Internet Things J 2(2):113-120 
Alzahrani A, Whitehead A (2015) Preprocessing realistic video for contactless heart rate monitoring using video magnification. In: Computer and robot vision (CRV), 2015 12th conference on, pp 261-268

Askew DA, Crossland L, Ware RS, Begg S, Cranstoun P, Mitchell P, Jackson CL (2012) Diabetic retinopathy screening and monitoring of early stage disease in general practice: design and methods. Contemp Clin Trials 33(5):969-975. doi:10.1016/j. cct.2012.04.011

Bai YW, Wu SC, Tsai CL (2012) Design and implementation of a fall monitor system by using a 3 -axis accelerometer in a smart phone. IEEE Trans Consum Electron 58(4):1269-1275

Baig MM, Gholamhosseini H (2013) Smart health monitoring systems: an overview of design and modeling. J Med Syst 37(2):9898. doi:10.1007/s10916-012-9898-z

Bauer K, Mendes LA (2015) Weblab of a control experiment in a newborn baby incubator. In: Remote engineering and virtual instrumentation (REV), 2015 12th international conference on, pp 163-71

Benelli G et al (2012) Health monitoring and wellness for all, a multichannel approach through innovative interfaces and systems. In: Proceedings of the 7th international conference on body area networks, BodyNets'12, ICST, Brussels, Belgium, Belgium: ICST (Institute for Computer Sciences, Social-Informatics and Telecommunications Engineering), pp 96-99. http://dl.acm.org/ citation.cfm?id=2442691.2442715

Bernacchia N et al (2014) Non contact measurement of heart and respiration rates based on kinect. In: Medical Measurements and Applications (MeMeA), 2014 IEEE International Symposium on, pp 1-5

Bifulco P et al (2014) Monitoring of respiration, seismocardiogram and heart sounds by a PVDF piezo film sensor. In: 20th IMEKO TC4 international symposium and 18th international workshop on ADC modelling and testing, pp 786-89. http://www.imeko. org/publications/tc4-2014/IMEKO-TC4-2014-285.pdf

Bisio I, Lavagetto F, Marchese M, Sciarrone A (2015a) Smartphonecentric ambient assisted living platform for patients suffering from co-morbidities monitoring. IEEE Commun Mag 53(1):34-41

Bisio I, Lavagetto F, Marchese M, Sciarrone A (2015b) A smartphonecentric platform for remote health monitoring of heart failure. Int J Commun Syst 28(11):1753-1771. doi:10.1002/dac.2778

Block VAJ, Pitsch E, Tahir P, Cree BAC, Allen DD, Gelfand JM (2016) Remote physical activity monitoring in neurological disease: a systematic review. PLOS One 11(4):1-41. doi:10.1371/journal. pone. 0154335

Bogdan G et al (2015) Remote assessment of heart rate by skin color processing. Communications and networking (BlackSeaCom), 2015 IEEE international black sea conference on, pp 112-116

Cao Y, Yang Y, Liu W (2012) E-FallD: a fall detection system using android-based smartphone. In: Fuzzy systems and knowledge discovery (FSKD), 2012 9th international conference on, pp 1509-13

Casacci P et al (2015) Alzheimer patient's home rehabilitation through ICT advanced technologies: the ALTRUISM project. In: Ambient assisted living: Italian forum 2014. Springer International Publishing, Cham, pp 377-385. doi:10.1007/978-3-319-18374-9_35

Chandrasekar D, Arnetz B, Levy P, Basu AS (2012) Plug-and-play, single-chip photoplethysmography. In: 2012 annual international conference of the IEEE engineering in medicine and biology society, pp 3243-46

Chen $\mathrm{M}$ et al (2014) A survey of recent developments in home M2M networks. IEEE Commun Surv Tutor 16(1):98-114
Cippitelli E, Fioranelli F, Gambi E, Spinsante S (2017) Radar and RGB-depth sensors for fall detection: a review. IEEE Sens J 17(12):3585-3604

Colantonio $S$ et al (2015) A smart mirror to promote a healthy lifestyle. Biosyst Eng 138:33-43

Couderc JP et al (2014) Pulse harmonic strength of facial video signal for the detection of atrial fibrillation. In: Computing in cardiology 2014, pp 661-664

Dhulipala VRS, Devadas P, Murthy PHST (2016) Mobile phone sensing mechanism for stress relaxation using sensor networks: a survey. Wirel Personal Commun 86(2):1013-1022. doi:10.1007/ s11277-015-2969-y

Donnelly N et al (2015) Development and integration of a surveillance monitoring solution to provide earlier detection of the deteriorating patient. In: 2015 37th Annual international conference of the IEEE engineering in medicine and biology society (EMBC), pp 1198-1202

Eastwood-Sutherland C, Gale TJ, Dargaville PA, Wheeler K (2014) Non-contact respiratory monitoring in neonates. In: Biomedical engineering international conference (BMEiCON), 2014 7th, pp $1-5$

Elfaramawy T, Fall CL, Morisette M, Lellouche F, Gosselin B (2017) Wireless respiratory monitoring and coughing detection using a wearable patch sensor network. In: 2017 15th IEEE International New Circuits and Systems Conference (NEWCAS), pp 197-200

El-Gayar O, Timsina P, Nawar N, Eid W (2013) Mobile applications for diabetes self-management: status and potential. J Diabetes Sci Technol 7(1):247-262. doi:10.1177/193229681300700130

Erden F, Velipasalar S, Alkar AZ, Cetin AE (2016) Sensors in assisted living: a survey of signal and image processing methods. IEEE Signal Process Mag 33(2):36-44

Faurholt-Jepsen M, Frost M, Vinberg M, Christensen EM, Bardram JE, Kessing LV (2014) Smartphone data as objective measures of bipolar disorder symptoms. Psychiatry Res 217(1):124-127. doi:10.1016/j.psychres.2014.03.009

Ferreira $\mathrm{F}$ et al (2015) Vital signs monitoring system using radio frequency communication: a medical care terminal for beddridden people support. Sens Transducers 185(2): 93-99. http:// www.sensorsportal.com/HTML/DIGEST/february_2015/ Vol_185/P_2607.pdf

Fioravanti A et al (2015) Automatic messaging for improving patients engagement in diabetes management: an exploratory study. Med Biol Eng Comput 53(12):1285-1294. doi:10.1007/ s11517-014-1237-8

Francis T, Madiajagan M, Kumar V (2015) Privacy issues and techniques in E-health systems. In: Proceedings of the 2015 ACM SIGMIS conference on computers and people research, SIGMIS-CPR'15, New York, NY, USA: ACM, pp 113-15. doi:10.1145/2751957.2751981

Ganesan M et al (2015) A novel based algorithm for the prediction of abnormal heart rate using bayesian algorithm in the wireless sensor network. In: Proceedings of the 2015 international conference on advanced research in computer science engineering technology (ICARCSET 2015), ICARCSET'15, New York, NY, USA: ACM, 53:1-53:5. doi:10.1145/2743065.2743118

Gibson RM et al (2016) Multiple comparator classifier framework for accelerometer-based fall detection and diagnostic. Appl Soft Comput 39:94-103

Giggins OM, Clay I, Walsh L (2017) Physical activity monitoring in patients with neurological disorders: a review of novel bodyworn devices. Dig Biomark 1(1):14-42

Gonzalez E et al (2015) Survey of WBSNs for pre-hospital assistance: trends to maximize the network lifetime and video transmission techniques ed. Aslam Nauman. Sens Basel Sens 15(5): 11993-12021 
González-López A, Ortega M, Penedo MG, Charlón P (2015) A webbased framework for anatomical assessment of the retina using \{OCT\}. Biosyst Eng 138:44-58

Greene S, Thapliyal H, Carpenter D (2016) IoT-based fall detection for smart home environments. In: 2016 IEEE international symposium on nanoelectronic and information systems (iNIS), pp 23-28. doi:10.1109/iNIS.2016.017

Guo H et al (2012) 2012 international workshop on information and electronics engineering sensor based real-time remote patient monitoring system: a study on mobile DB construction of minimum network traffic in use of HTML5 WebSQL. Procedia Eng 29:2382-2387

Hassan MA, Malik AS, Fofi D, Saad N, Karasfi B, Ali YS, Meriaudeau F (2017) Heart rate estimation using facial video: a review. Biomed Signal Process Control 38(Supplement C). doi:10.1016/j.bspc.2017.07.004

Hazenberg CEVB, Bus SA, Kottink AIR, Bouwmans CAM, Schönbach-Spraul AM, van Baal SG (2012) Telemedical home-monitoring of diabetic foot disease using photographic foot imaginga feasibility study. J Telemed Telecare 18(1):32-36. doi:10.1258/ jtt.2011.110504

Hegde R et al (2013) Technical advances in fall detection system-a review. Int J Comput Sci Mobile Comput 2(7): 152-60. http:// www.ijcsmc.com/docs/papers/July2013/V2I7201334.pdf

Hood M, Wilson R, Corsica J, Bradley L, Chirinos D, Vivo A (2016) What do we know about mobile applications for diabetes selfmanagement? A review of reviews. J Behav Med 39:981-994

Hossain MA, Ahmed DT (2012) Virtual caregiver: an ambient-aware elderly monitoring system. IEEE Trans Inf Technol Biomed 16(6):1024-1031

Hu S, Azorin-Peris V, Zheng J (2013) Opto-physiological modeling applied to photoplethysmographic cardiovascular assessment. J Healthc Eng 4(4): 505-528. http://downloads.hindawi.com/ journals/jhe/2013/705820.pdf

Igual R, Medrano C, Plaza I (2013) Challenges, issues and trends in fall detection systems. BioMed Eng OnLine 12(1):1-24. doi:10.1186/1475-925X-12-66

Janssen R, Wang W, Moço A, de Haan G (2016) Video-based respiration monitoring with automatic region of interest detection. Physiol Meas 37(1): 100. http://stacks.iop.org/0967-3334/37/ $\mathrm{i}=1 / \mathrm{a}=100$

Karakostas A et al (2015) A sensor-based framework to support clinicians in dementia assessment: the results of a pilot study. In: ambient intelligence-software and applications: 6th international symposium on ambient intelligence (ISAmI 2015), eds. Amr Mohamed et al. Springer International Publishing, Cham, pp 213-21. doi:10.1007/978-3-319-19695-4_22

Karan O, Bayraktar C, Gümüşkaya H, Karlık B (2012) Diagnosing diabetes using neural networks on small mobile devices. Expert Syst Appl 39(1):54-60

Karlen W et al (2014) Respiratory rate assessment from photoplethysmographic imaging. In: 2014 36th annual international conference of the IEEE engineering in medicine and biology society, pp 5397-5400

Khan SS, Hoey J (2017). Review of fall detection techniques: a data availability perspective. Med Eng Phys 39(Supplement C):12-22. doi:10.1016/j.medengphy.2016.10.014

Khattak HA, Ruta M, Di Sciascio E, Sciascio D (2014) CoAP-based healthcare sensor networks: a survey. In: Proceedings of 2014 11th international Bhurban conference on applied sciences technology (IBCAST) Islamabad, Pakistan, 14th-18th January, 2014, pp 499-503

Kozlovszky M, Kovacs L, Karoczkai K (2015) Cardiovascular and diabetes focused remote patient monitoring. In: Braidot A, Hadad A (eds) VI Latin American congress on biomedical engineering CLAIB 2014, Paran\{á\}, Argentina 29, 30 \{\&\} 31 October
2014. Springer International Publishing, Cham, pp 568-71. doi:10.1007/978-3-319-13117-7_145

Kumar DJRK, Kotnana N (2012) Design and implementation of portable health monitoring system using PSoC mixed signal array chip. IJRTE 1(3): 59-65. http://docplayer.net/7620189-Designand-implementation-of-portable-health-monitoring-systemusing-psoc-mixed-signal-array-chip.html

Lakshminarayanan V, Zelek J, McBride A (2015) 'Smartphone Science' in eye care and medicine. Opt Photon News: 44-51. http://www. osa-opn.org/home/articles/volume_26/january_2015/features/ smartphone_science\%E2\%80\%9D_in_eye_care_and_medicine/

Lam K-Y et al (2015) Activity tracking and monitoring of patients with Alzheimer's disease. Multimed Tools Appl. doi:10.1007/ s11042-015-3047-x

Lanata A et al (2015) Complexity index from a personalized wearable monitoring system for assessing remission in mental health. IEEE J Biomed Health Inf 19(1):132-139

Ling THY, Wong LJ, Tan JEH, Kiu KY (2015) Non-intrusive human body temperature acquisition and monitoring system. In: 2015 6th international conference on intelligent systems, modelling and simulation, pp 16-20

Lukáč T, Púčik J, Chrenko L (2014) Contactless recognition of respiration phases using web camera. In: Radioelektronika (RADIOELEKTRONIKA), 2014 24th international conference, pp 1-4

Luprano J et al (2013) heartcycle: advanced sensors for telehealth applications. In: 2013 35th annual international conference of the IEEE engineering in medicine and biology society (EMBC), pp 6984-87

Ly TT, Breton MD, Keith-Hynes P, De Salvo D, Clinton P, Benassi K et al (2014) Overnight glucose control with an automated, unified safety system in children and adolescents with type 1 diabetes at diabetes camp. Diabetes Care 37(8):2310-2316. doi:10.2337/ dc14-0147

Magno M et al (2013) A versatile biomedical wireless sensor node with novel drysurface sensors and energy efficient power management. In: Advances in sensors and interfaces (IWASI), 2013 5th IEEE international workshop on, pp 217-22

Mainanwal V, Gupta M, Upadhayay SK (2015) A survey on wireless body area network: security technology and its design methodology issue. In: Innovations in information, embedded and communication systems (ICIIECS), 2015 international conference on, pp 1-5

Malhi K et al (2012) A zigbee-based wearable physiological parameters monitoring system. IEEE Sens J 12(3):423-430

Mastorakis G, Makris D (2014) Fall detection system using kinect's infrared sensor. J Real Time Image Process 9(4):635-646. doi:10.1007/s11554-012-0246-9

McDuff DJ, Estepp JR, Piasecki AM, Blackford EB (2015) A survey of remote optical photoplethysmographic imaging methods. In: 2015 37th annual international conference of the IEEE engineering in medicine and biology society (EMBC), pp 6398-6404

Mishra A, Agrawal DP (2015) Continuous health condition monitoring by 24/7 sensing and transmission of physiological data over 5-G cellular channels. In: Computing, networking and communications (ICNC), 2015 international conference on, pp 584-90

Mishra D, Gogna G, Barsaiyan A, Sarkar M (2015) "Blood Pulsation Measurement Using Linearly Polarized Light". IEEE Sens J 15(8):4488-4495

Mobasheri MH et al (2015) The uses of smartphones and tablet devices in surgery: a systematic review of the literature. Surg 158(5):1352-1371

Monkaresi H, Calvo RA, Yan H (2014) A machine learning approach to improve contactless heart rate monitoring using a webcam. IEEE J Biomed Health Inf 18(4):1153-1160 
Mubashir M, Shao L, Seed L (2013). A survey on fall detection: principles and approaches. Neurocomputing 100(Supplement C):144152. doi:10.1016/j.neucom.2011.09.037

Nadeem A et al (2015) Application specific study, analysis and classification of body area wireless sensor network applications. Comput Netw 83:363-380

Naranjo-Hernandez D, Roa LM, Reina-Tosina J, Estudillo-Valderrama MA (2012) Personalization and adaptation to the medium and context in a fall detection system. IEEE Trans Inf Technol Biomed 16(2):264-271

Naslund JA, Marsch LA, McHugo GJ, Bartels SJ (2015) Emerging mHealth and eHealth interventions for serious mental illness: a review of the literature. J Mental Health 24(5):321-332. doi:10. 3109/09638237.2015.1019054

Nguyen HH, Silva JNA (2016) Use of smartphone technology in cardiology. Trends Cardiovasc Med 26(4):376-386

O'Grady MJ, Retterath AJ, Keenan DB, Kurtz N (2012) The use of an automated, portable glucose control system for overnight glucose control in adolescents and young adults with type 1 diabetes. Diabetes Care 35(11):2182-2187

Otoom AF et al (2015) Effective diagnosis and monitoring of heart disease. Int J Softw Eng Appl 9(1): 143-156. http://www.sersc. org/journals/IJSEIA/vol9_no1_2015/12.pdf

Paoli R et al (2012) A system for ubiquitous fall monitoring at home via a wireless sensor network and a wearable mote. Expert Syst Appl 39(5):5566-5575

Papon MTI, Ahmad I, Saquib N, Rahman A (2015) Non-invasive heart rate measuring smartphone applications using on-board cameras: a short survey. In: Networking systems and security (NSysS), 2015 international conference on, pp 1-6

Parnandi A, Gutierrez-Osuna R (2013) Contactless measurement of heart rate variability from pupillary fluctuations. In: Affective computing and intelligent interaction (ACII), 2013 humaine association conference on, pp 191-196

Patel S et al (2012) A review of wearable sensors and systems with application in rehabilitation. J NeuroEng Rehab 9(1):1-17. doi:10.1186/1743-0003-9-21

Pereira $\mathrm{T}$ et al (2013) Comparison of low-cost and noninvasive optical sensors for cardiovascular monitoring. IEEE Sens J 13(5):1434-1441

Pinheiro EC, Postolache OA, Girão PS (2013) Dual architecture platform for unobtrusive wheelchair user monitoring. In: Medical measurements and applications proceedings (MeMeA), 2013 IEEE international symposium on, pp 124-29

Prabhakar SK, Rajaguru H (2017) Development of patient remote monitoring system for epilepsy classification. In: Goh J, Lim CT, Leo HL (eds) The 16th international conference on biomedical engineering: ICBME 2016, 7th to 10th December 2016, Singapore. Singapore: Springer Singapore, pp 80-87. doi:10.1007/978-981-10-4220-1_16

Price E, Moore G, Galway L, Linden M (2015) Towards a mobile assistive technology for monitoring and assessing cognitive fatigue in individuals with acquired brain injury. In: Computer and information technology; ubiquitous computing and communications; dependable, autonomic and secure computing; pervasive intelligence and computing (CIT/IUCC/DASC/PICOM), 2015 IEEE international conference on, pp 1487-91

Ramesh MV, Anand S, Rekha P (2012) A mobile software for health professionals to monitor remote patients. In: 2012 Ninth international conference on wireless and optical communications networks (WOCN), pp 1-4

Rasmussen BSB et al (2015) A qualitative study of the key factors in implementing telemedical monitoring of diabetic foot ulcer patients. Int J Med Inf 84(10):799-807

Ricci RP et al (2013) Effectiveness of remote monitoring of CIEDs in detection and treatment of clinical and device-related cardiovascular events in daily practice: the homeguide registry. Europace 15(7):970-977

Rodbard D (2016) Continuous glucose monitoring: a review of successes, challenges, and opportunities. Diabetes Technol Therapeut 18(S2), S2-3-S2-13. doi:10.1089/dia.2015.0417

Rodríguez AM, Castro JR (2015) Pulse rate variability analysis by video using face detection and tracking algorithms. In: 2015 37th Annual international conference of the ieee engineering in medicine and biology society (EMBC), pp 5696-5699

Rotariu C et al (2015) A wireless sensor network for remote monitoring of bioimpedance. In: 2015 38th international spring seminar on electronics technology (ISSE), pp 487-490

Sannino G, De Falco I, De Pietro G (2015) A supervised approach to automatically extract a set of rules to support fall detection in an mhealth system. Appl Soft Comput 34: 205-216. http://www. sciencedirect.com/science/article/pii/S1568494615002975

Sardini E, Serpelloni M, Pasqui V (2015) Wireless wearable T-shirt for posture monitoring during rehabilitation exercises. IEEE Trans Instrum MeasMeas 64(2):439-448

Sathyanarayana S, Satzoda RK, Sathyanarayana S, Thambipillai S (2015) Vision-based patient monitoring: a comprehensive review of algorithms and technologies. J Ambient Intell Hum Comput. doi:10.1007/s12652-015-0328-1

Sawand A, Djahel S, Zhang Z, Naït-Abdesselam F (2015) Toward energy-efficient and trustworthy eHealth monitoring system. China Commun 12(1):46-65

Schneider RB, Biglan KM (2017) The promise of telemedicine for chronic neurological disorders: the example of Parkinson's disease. Lancet Neurol 16(7):541-551. doi:10.1016/ S1474-4422(17)30167-9

Serhani MA, El Menshawy M, Benharref A (2016) SME2EM: smart mobile end-to-end monitoring architecture for life-long diseases. Comput Biol Med 68:137-154

Shakshuki E, Almadani B, Bin-Yahya M, Shakshuki EM (2015) The 6th international conference on emerging ubiquitous systems and pervasive networks (EUSPN 2015)/the 5th international conference on current and future trends of information and communication technologies in healthcare (ICTH-2015)/ affiliated workshops E-AMBULANCE: real-time integration platform for heterogeneous medical telemetry system. Procedia Comput Sci 63:400-407. http://www.sciencedirect.com/ science/article/pii/S1877050915024941

Shao D et al (2016) Noncontact monitoring of blood oxygen saturation using camera and dual-wavelength imaging system. IEEE Trans Biomed Eng 63(6):1091-1098

Sharma M, Barbosa K, Ho V, Griggs D, Ghirmai T, Krishnan SK et al (2017) Cuff-less and continuous blood pressure monitoring: a methodological review. Technologies 5(2):21

Silva BMC et al (2015) Mobile-health: a review of current state in 2015. J Biomed Inf 56:265-272. http://www.sciencedirect. com/science/article/pii/S1532046415001136

Sola-Valls N et al (2015) Telemedicine for monitoring MS activity and progression. Curr Treat Opt Neurol 17(11):1-13. doi:10.1007/s11940-015-0377-y

Sorwar G, Hasan R (2012) Smart-TV based integrated e-health monitoring system with agent technology. In: Advanced information networking and applications workshops (WAINA), 2012 26th international conference on, pp 406-11

Sugita N et al (2015) Techniques for estimating blood pressure variation using video images. In: 2015 37th annual international conference of the IEEE engineering in medicine and biology society (EMBC), pp 4218-21

Suh $\mathrm{M}$ et al (2012) Dynamic self-adaptive remote health monitoring system for diabetics. In: 2012 Annual international conference of the ieee engineering in medicine and biology society, pp 2223-26 
Szydlo T, Koneiczny M (2015) Mobile devices in the open and universal system for remote patient monitoring. IFAC-PapersOnLine 48(4):296-301

Tanantong T, Nantajeewarawat E, Thiemjarus S (2015) False alarm reduction in bsn-based cardiac monitoring using signal quality and activity type information. Sensors 15(2): 3952. http://www. mdpi.com/1424-8220/15/2/3952

Tarassenko L, Villarroel M, Guazzi A, Jorge J, Clifton DA, Pugh C (2014) Non-contact video-based vital sign monitoring using ambient light and auto-regressive models. Physiol Meas 35(5):807. http://stacks.iop.org/0967-3334/35/i=5/a=807

Thakur RK, Anoop CS (2015) A non-contact capacitance based electrocardiograph and associated heart-rate detection using enhanced fourier interpolation method. In: 2015 37th annual international conference of the IEEE engineering in medicine and biology society (EMBC), pp 849-52

Thelen $\mathrm{S}$ et al (2015) Using off-the-shelf medical devices for biomedical signal monitoring in a telemedicine system for emergency medical services. IEEE J Biomed Health Inf 19(1):117-123

Tsakalakis M, Bourbakis NG (2014) Health care sensor based systems for point of care monitoring and diagnostic applications: a brief survey. In: 2014 36th annual international conference of the IEEE engineering in medicine and biology society, pp 6266-69

Vashist SK (2012) Non-invasive glucose monitoring technology in diabetes management: a review. Anal Chim Acta 750:16-27. doi:10.1016/j.aca.2012.03.043

Wac K, Tsiourti C (2014) Ambulatory assessment of affect: survey of sensor systems for monitoring of autonomic nervous systems activation in emotion. IEEE Trans Affect Comput 5(3):251-272
Wang C-S, Liu C-W, Wang T-H (2013) A remote health care system combining a fall down alarm and biomedical signal monitor system in an android smart-phone. Int J Adv Comput Sci Appl 4(9): 185-88. http://citeseerx.ist.psu.edu/viewdoc/download?doi=10. 1.1.404.450\&rep $=$ rep1\&type $=$ pdf

Wang Q et al (2015a) Smart rehabilitation garment for posture monitoring. In: 2015 37th Annual international conference of the IEEE engineering in medicine and biology society (EMBC), pp 5736-39

Wang Y, Kung L, Ting C, Byrd TA (2015b) Beyond a technical perspective: understanding big data capabilities in health care. In: System sciences (HICSS), 2015 48th Hawaii international conference on, pp 3044-53

$\mathrm{Yu}$ M et al (2012) A posture recognition-based fall detection system for monitoring an elderly person in a smart home environment. IEEE Trans Inf Technol Biomed 16(6):1274-1286

Zhan A et al (2016) High frequency remote monitoring of Parkinson's disease via smartphone: platform overview and medication response detection. http://arxiv.org/abs/1601.00960

Zhang S et al (2015) Design of a novel telerehabilitation system with a force-sensing mechanism ed. Vittorio MN, Passaro. Sens Basel Sens 15(5):11511-11527

Zhu Z et al (2015) Wearable sensor systems for infants. Sensors 15(2): 3721. http://www.mdpi.com/1424-8220/15/2/3721 